\title{
Quasi Optical Multi-Ray Model For Wireless Communication Link in Millimeter Wavelengths
}

\author{
Liat Rapaport $^{1, *}$, Ariel Etinger ${ }^{1}$, Boris Litvak ${ }^{1}$, Gad $_{\text {Pinhasi }}{ }^{2}$, and Yosef Pinhasi $^{1}$ \\ ${ }^{1}$ Ariel University, Dept. of Electrical and Electronics Engineering \\ ${ }^{2}$ Ariel University, Dept. of Chemical Engineering
}

\begin{abstract}
The spectrum of millimeter waves lay above $30 \mathrm{GHz}$. The band between $30 \mathrm{GHz}$ up to $300 \mathrm{GHz}$ is called Extremely High Frequencies (EHF). This wide spectrum is relatively free of users and have recently became relevant for realizations of wireless communications an radars, including the fifth generation (5G) of cellular communications. Due to the short wavelengths, the propagation of millimeter waves can be analyzed using quasi-optical ray techniques. We present a multi-ray analysis of millimeter wave wireless link in the presence of multipath. The analysis is applicable for indoor and outdoor links and considers reflections from walls and buildings. It is shown that line-of-sight is not necessarily required in scenarios where multiple reflections exist as in long corridors, canyons and tunnels. The theoretical results are verified experimentally with a link in the W-band $(94 \mathrm{GHz})$
\end{abstract}

\section{Introduction}

Indoor communication link suffers from obstacles that can disturb the signal to travel straight from the transmitter to the receiver. When the transmitted signal strikes those obstacles it can be reflected, diffracted, or scattered. The reflected signals create additional paths in addition to the line-of-sight (LOS) one. The signals, arriving from the receiver in different paths, combine coherently causing destructive or constructive interference frequency selective effects.

In short wavelengths it is easy to analyze the paths using quasi-optical techniques such as ray tracing method [1]. The Two Ray Model is applied in a simple scenario when the transmitter and the receiver are placed above ground at outdoor environment and the signal is reflected to the receiver only from the ground. At that scenario the receiver sums up only two signals; the LOS signal and the ground reflected signal. Multi-ray Model is used in an indoor environment, where several reflections occur from obstacles, surfaces, walls, ceiling and floor.

Interesting phenomena emerge when the communication link is in narrow corridors or in tunnels. Such effects may arise also in outdoor links operating in long canyon environment. Those scenarios can be treated as extremely long rooms where the front and back walls are far away from the transmitter and receiver. Several methods have been proposed to describe wave propagation in tunnel. Their accuracy is mainly determined by the dimensions of the space relative to the wavelengths. Ray tracing become relevant when the wavelength is much shorter than the dimensions of the tunnel or the canyon. It uses Geometrical Optics (GO) approach to describe the signal strength

\footnotetext{
*Corresponding author: liatra@ariel.ac.il
}

at every point along the propagation path. This method can be easily applied when the communication is carried in short wavelength, like millimeter waves. The link inside a tunnel are composed of the LOS path, the reflected signals from top and bottom surfaces and the reflected signals from walls surfaces. The radio wave characteristics change by the tunnel structure and by it internal curvature [2-5].

In this study we investigate the implementation of the ray tracing model in wireless links operating in millimeter waves. We demonstrate the analysis by performing set of experiments. Comparison is made with simulation results. We found a reasonable correspondence between our model and our lab results.

\section{Millimeter Wave Reflection From Dielectric Surfaces}

First we examine the reflections from walls as dielectric surfaces. It is important to identify how the dielectric permittivity of the building material affects the reflection coefficient. We use the Fresnel's equations for the reflection coefficient, differing between the TE polarization and the TM polarization:

$$
\begin{gathered}
\Gamma_{T M_{i}}=\frac{\epsilon_{r} \sin \left(\theta_{g}\right)-\sqrt{\epsilon_{r}-\cos ^{2}\left(\theta_{g}\right)}}{\epsilon_{r} \sin \left(\theta_{g}\right)+\sqrt{\epsilon_{r}-\cos ^{2}\left(\theta_{g}\right)}} \\
\Gamma_{T E_{i}}=\frac{\sin \left(\theta_{g}\right)-\sqrt{\epsilon_{r}-\cos ^{2}\left(\theta_{g}\right)}}{\sin \left(\theta_{g}\right)+\sqrt{\epsilon_{r}-\cos ^{2}\left(\theta_{g}\right)}}
\end{gathered}
$$


Here $\theta_{g}$ is the grazing angle between the incident ray and the surface [6]. This angle is a result of the transmitter and receiver locations and the distance between them. $\epsilon_{r}$ is the relative dielectric coefficient of the scattering material. It is a frequency dependent quantity. Table 1 summarizes dielectric coefficients of different materials at W-band [7, 8].

Table 1. Relative dielectric coefficients of materials in Wband frequencies

\begin{tabular}{|c|c|c|}
\hline Material & Dielectric Constant $\epsilon_{r}$ & Frequency \\
\hline Alumina & 3.1 & $100 \mathrm{GHz}$ \\
\hline Soil (dry) & 2.9 & $94 \mathrm{GHz}$ \\
\hline Soil (moist) & 3.7 & $94 \mathrm{GHz}$ \\
\hline Nylon & 2.993 & $100 \mathrm{GHz}$ \\
\hline Asphalt & 3.18 & $94 \mathrm{GHz}$ \\
\hline Formica & $4-6$ & - \\
\hline Concrete & 4.5 & - \\
\hline
\end{tabular}

We calculated the reflection coefficient for different values of dielectric coefficients for both polarizations as a function of distance between the receiver and the transmitter (See Figure 3). Inspection the graphs in Fig. 1 reviles a small dependence on the material properties.

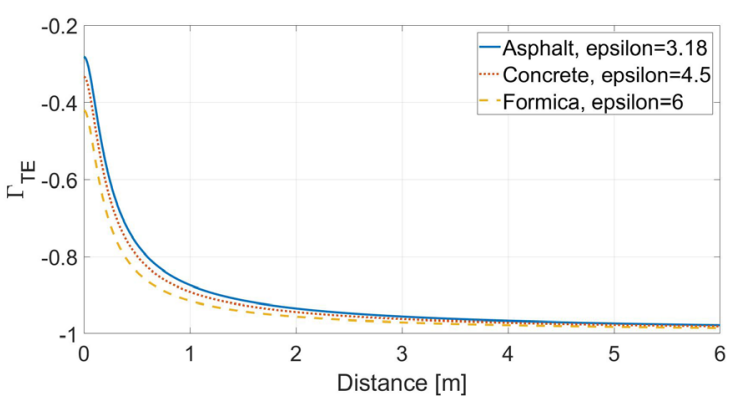

(a) TE

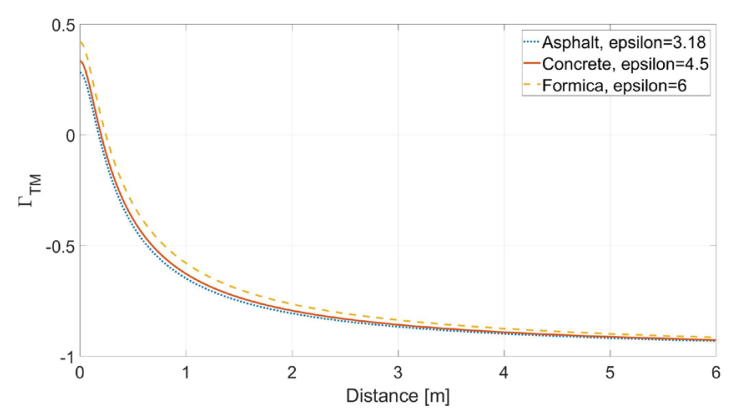

(b) TM

Fig. 1. Reflection coefficient from different materials

It is important to note that the Fresnel equations assume that the scattering surface is a 'specular' reflector. This is true if the surface roughness follows the Rayleigh criterion, requiring that the phase shift $\Delta \phi$ caused by the changes in the surface, is smaller than a value leading to a destructive interference, i.e. $\Delta \phi<\pi / 2$. This leads to an explicit expression for roughness measure $\Delta h<\frac{\lambda}{8 \sin \theta_{g}}$.

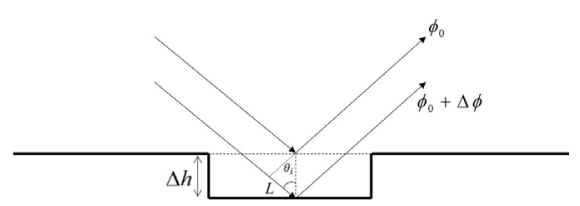

Fig. 2. Phase shift caused by height differences diagram

\section{Two Rays Above Ground}

We start with a simple scenario where only one additional path exists, besides to the direct line-of-sight. An example for this path is a reflection from ground. Two rays arrive at the receiver, as shown in Figure 3. The link budget for this case is calculated via the summation:

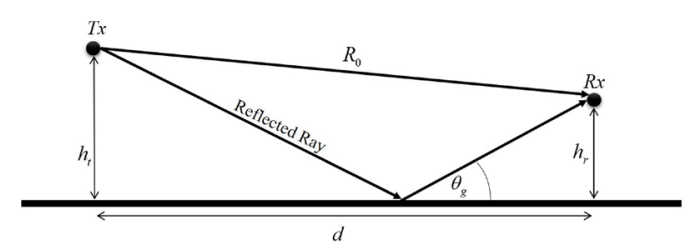

Fig. 3. Two rays diagram

$$
\frac{P_{r}}{P_{t}}=\left(\frac{\lambda}{4 \pi}\right)^{2} \cdot\left|\frac{G_{d}\left(\theta_{0}\right) e^{-j k R_{0}}}{R_{0}}+\frac{G_{d}\left(\theta_{g}\right) \Gamma_{0} e^{-j k R_{p}}}{R_{p}}\right|^{2}
$$

$P_{t}:$ Transmitter power

$P_{r}:$ Received power

$\lambda$ : Wavelength

$R_{0}$ : LOS path length between $T_{x}$ and $R_{x}$

$R_{p}$ : Reflected ray path length between $T_{x}$ and $R_{x}$

$k$ : Wavenumber, $k=2 \pi / \lambda$

$G_{t}, G_{r}$ : Gain of $T_{x}$ and $R_{x}$ antennas

$G_{d}\left(\theta_{g}\right)=\sqrt{G_{t}(\theta) \cdot G_{r}(\theta)}$ : Geometric mean of $T_{x}$ and $R_{x}$ antenna gains at $\theta_{g}$ angle

$\Gamma_{0}=$ Reflection coefficient

If the transmitter and the receiver are located at same heights above the reflecting surface, i.e. $h_{t}=h_{r}$, and both transmitting and receiving antennas are directed to each other, the total gain is $G_{d}\left(\theta_{0}\right)=G_{0}$. Defining $F\left(\theta_{g}\right)=\frac{G_{d}\left(\theta_{g}\right)}{G_{0}}$, the path loss can be written in a normalized expression [9]:

$$
\frac{P_{r}(\text { Two }- \text { rays })}{P_{r}(L O S)}=\left|1+\frac{R_{0}}{R_{p}} \cdot F\left(\theta_{g}\right) \cdot \Gamma_{0} \cdot e^{-j k\left(R_{p}-R_{0}\right)}\right|^{2}
$$

In millimeter wave communication links, directive antennas are usually employed. In our demonstration we use 
a pyramidal horn antenna, shown in Figure 4a, with a gain of $G_{0}=24 \mathrm{dBi}$. Its radiation pattern was calculated using the formulation given in $[10,11]$.

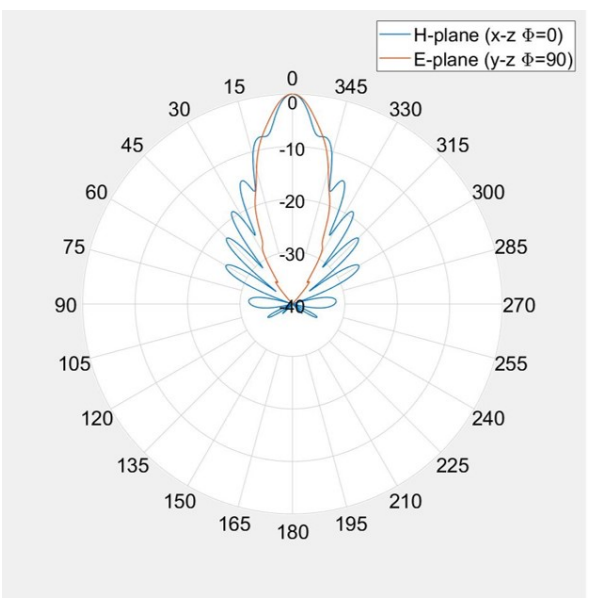

(a) Horn radiation pattern

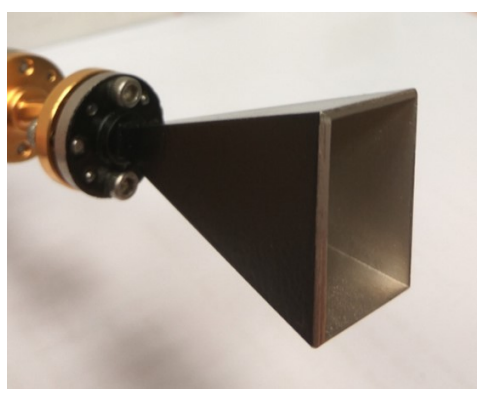

(b) Horn antenna

Fig. 4. Horn antenna specifications

An experiment was carried out to verify the theoretical analysis. Figure 5 shows the experimental setup. We use a W-band, continuous wave, solid state source operating at $94 \mathrm{GHz}$ as the transmitter. Its power is $17 \mathrm{dBm}$. The receiver is based on an zero bias schottky detector. The transmitter and receiver were both places $10 \mathrm{~cm}$ above a flat table covered with Formica (dielectric coefficient of $\left.\epsilon_{r}=6\right)$.

Figure 6 shows the correspondence between the theoretical calculations and the experimental results.

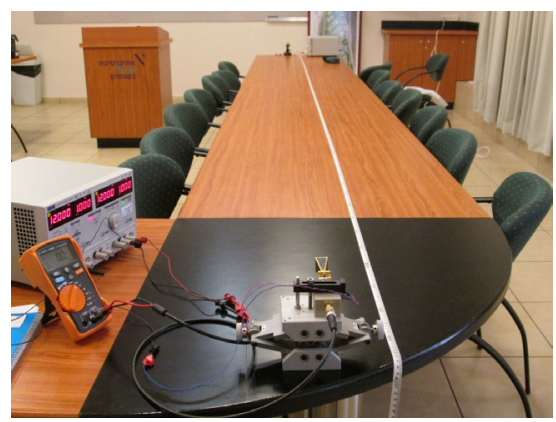

Fig. 5. Two rays setup experiment at $94 \mathrm{GHz}$

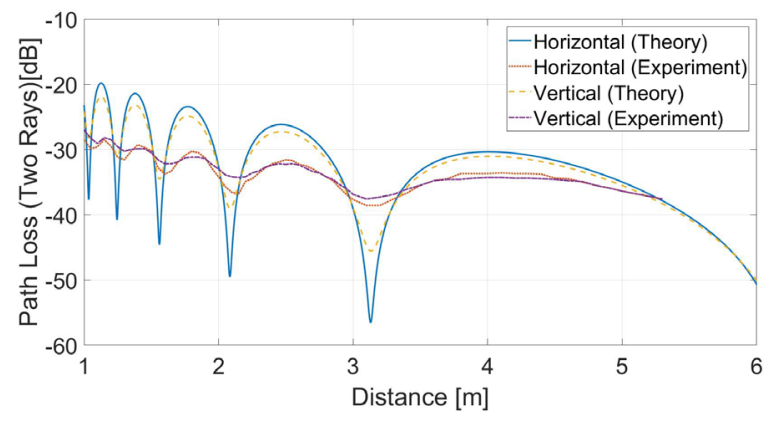

Fig. 6. Comparison between two rays simulation and experiments results at $94 \mathrm{GHz}$

\section{Propagation In Tunnels}

Ray tracing model in tunnels is divided into two basic approaches for calculating the rays paths; Shooting and Bouncing Ray (SBR) techniques and the image method. The latter uses geometrical optics (GO) to follow the paths along the tunnel. The received signal is a result of of three types of arriving waves; LOS path, vertical paths (diffracted from the top and bottom surfaces) and the horizontal waves (diffracted from the walls) [12-15]

The basics of ray tracing is based on Friss equation combining with Fresnel's coefficient to predict the multipath link. The polarization of the transmitter antenna is an important parameter, since it determines the type of reflection (TE or TM) from each surface. For vertically polarized antenna, the waves strikes the walls will have a reflection coefficient of the TM polarization and the waves strikes the top and bottom surfaces will have the TE reflection coefficient. As expected, an horizontally polarized antenna will produce an opposite response; TM reflections will occur from the top and bottom surfaces, while the reflection from the walls will be of the TE type. Table 2 summarizes reflection features at each transmitting antenna polarization.

Table 2. Reflection coefficients polarization

\begin{tabular}{|l|l|l|}
\hline $\begin{array}{l}\text { Antenna polar- } \\
\text { ization }\end{array}$ & $\begin{array}{l}\text { Walls polariza- } \\
\text { tion }\end{array}$ & $\begin{array}{l}\text { Floor and ceiling } \\
\text { polarization }\end{array}$ \\
\hline Vertical & $\Gamma_{T E}$ & $\Gamma_{T M}$ \\
\hline Horizontal & $\Gamma_{T M}$ & $\Gamma_{T E}$ \\
\hline
\end{tabular}

$$
\begin{aligned}
\frac{P_{r}}{P_{t}}=\left(\frac{\lambda}{4 \pi}\right)^{2} \cdot \mid \frac{G_{d} e^{-j k R_{0}}}{R_{0}} & +2 \sum_{i=1}^{n_{v}} \frac{G_{d}(\theta)\left(\Gamma_{i}\right)^{i} e^{-j k R_{i}}}{R_{i}} \\
& +\left.2 \sum_{i=1}^{n_{h}} \frac{G_{d}(\theta)\left(\Gamma_{i}\right)^{i} e^{-j k R_{i}}}{R_{i}}\right|^{2}
\end{aligned}
$$

Equation (5) describes the link budget taking into account multiple reflections in a tunnel. For simplicity it is 


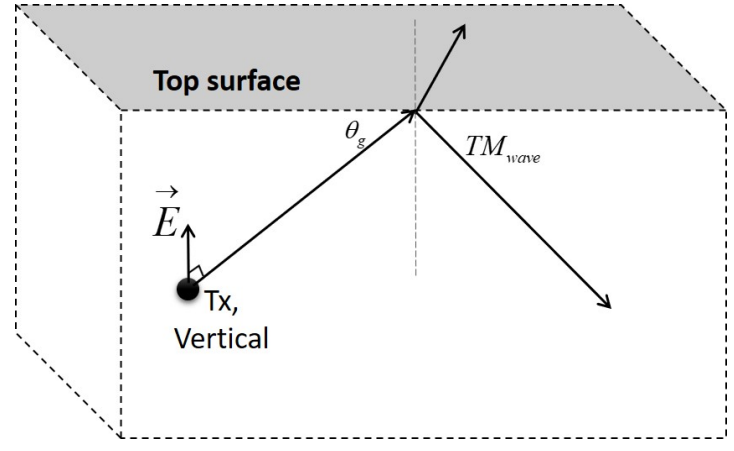

(a) TM reflection

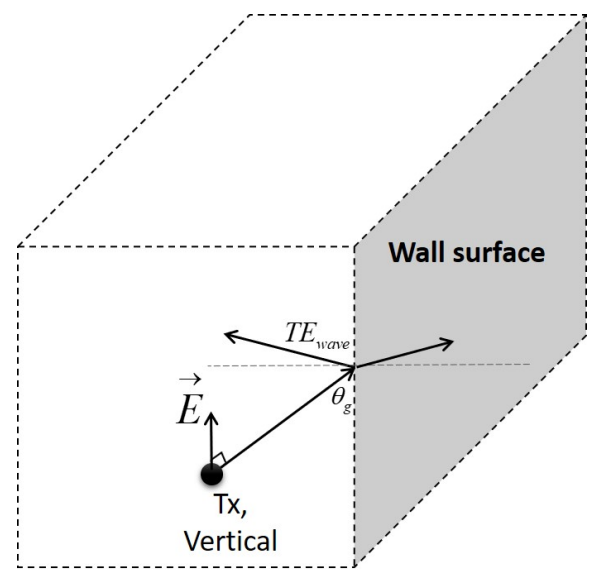

(b) TE reflection

Fig. 7. Polarization in tunnel propagation

assumed that $h_{t}=h_{r}=0.5 \mathrm{H}$ and $w_{t}=w_{r}=0.5 \mathrm{~W}$. The factor 2 appears in Eq.(5) derived from the symmetric scenario when both rays from ceiling and floor or from the two walls arrives with the same power and phase to the detector. A normalized presentation of equation (5) is:

$$
\begin{array}{r}
\frac{P_{r}(\text { Multi-ray })}{P_{r}(\text { LOS })}=\mid 1+2 \sum_{i=1}^{n_{v}} F\left(\theta_{g}\right) \cdot\left(\Gamma_{i}\right)^{i} \frac{R_{0}}{R_{i}} \cdot e^{-j k\left(R_{i}-R_{0}\right)} \\
+\left.2 \sum_{i=1}^{n_{h}} F\left(\theta_{g}\right) \cdot\left(\Gamma_{i}\right)^{i} \frac{R_{0}}{R_{i}} \cdot e^{-j k\left(R_{i}-R_{0}\right)}\right|^{2}
\end{array}
$$

Calculations of the reflected ray paths $R_{i}$ were done using image ray tracing [16] (see Figure 8). The resulted path lengths are given by:

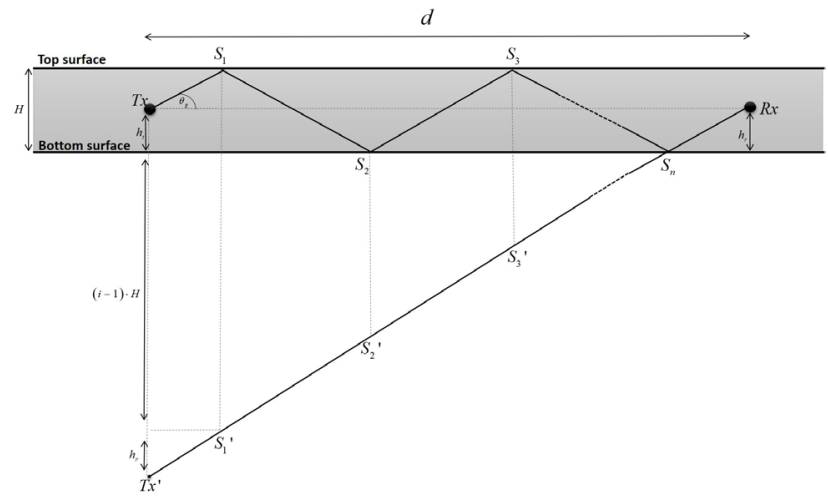

Fig. 8. Reflected rays using images

$$
R_{i}=\sqrt{d^{2}+(i H)^{2}}
$$

while their respective grazing angles are found from:

$$
\theta_{g}=\tan ^{-1}\left(\frac{i H}{d}\right)
$$

In order to carry an experimental measurement of the path loss along a tunnel, a small tunnel model made of wood coated with Formica was constructed, as shown in Figure 10. The experiment was done at $94 \mathrm{GHz}$, where the antennas of both transmitter and receiver were pyramidal horns with a gain of $24 \mathrm{dBi}$, as in Figure $4 \mathrm{a}$. The horns were placed in the center of the tunnel.

A comparison between numerical calculations using (5) and experimental results are presented in Fig.9 for vertical and horizontal polarizations.

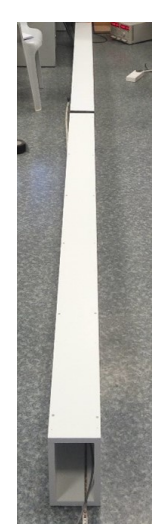

Fig. 9. Lab experiment tunnel

\section{Summary and Conclusions}

The spectrum above $30 \mathrm{GHz}$ is becoming a candidate for realization of wireless communication links. This millimeter wave portion of the electromagnetic spectrum is relatively free of users and being considered for wide band data transfer. In such links, multi-path effects emerge mainly due to scattering from objects and surfaces.

The small wavelength enables utilization of quasioptical techniques for the analysis of the link budget in 


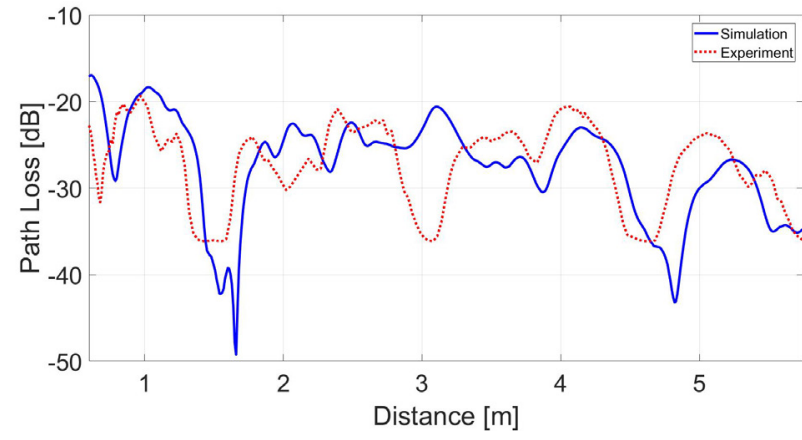

(a) Vertical polarization

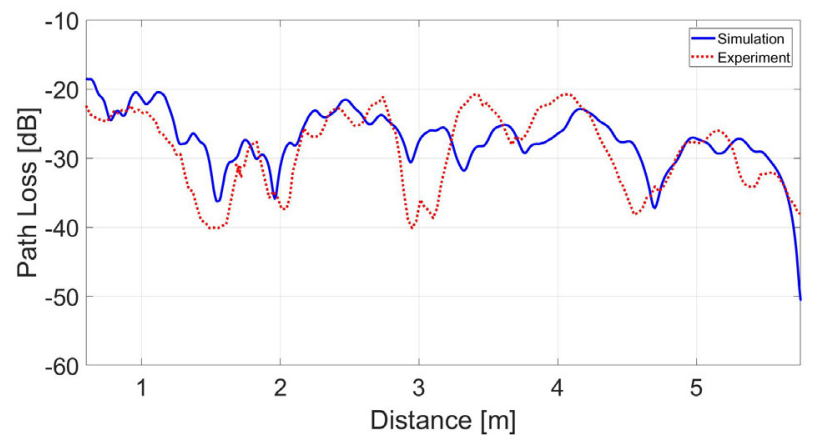

(b) Horizontal polarization

Fig. 10. Comparison between simulation to experiment results at $94 \mathrm{GHz}$

indoor and outdoor scenarios. A theoretical study of the multi-path caused by a single reflection and multiple reflections is presented and analyzed using the two- and multi- ray models. Experimental verification of the theory demonstrates that the ray models can estimate the performances of a wireless link operating in millimeter waves accurately also in tunnels where multiple reflections are involved.

\section{References}

1. Goldsmith, A. Wireless communications (Cambridge university press, 2005).

2. Collin, R. E. Antennas and radiowave propagation (McGraw-Hill, 1985).

3. Kermani, M. H. \& Kamarei, M. A ray-tracing method for predicting delay spread in tunnel environments in Personal Wireless Communications, 2000 IEEE International Conference on (2000), $538-542$.
4. Kim, Y., Jung, M. \& Lee, B. Analysis of radio wave propagation characteristics in rectangular road tunnel at $800 \mathrm{MHz}$ and $2.4 \mathrm{GHz}$ in Antennas and Propagation Society International Symposium, 2003. IEEE 3 (2003), 1016-1019.

5. Zhang, Y., Liu, Y., Sun, J., Wang, C.-X. \& Ge, X. Impact of Different Parameters on Channel Characteristics in a High-Speed Train Ray Tracing Tunnel Channel Model in Vehicular Technology Conference (VTC Spring), 2017 IEEE 85th (2017), 1-5.

6. Rappaport, T. S. et al. Wireless communications: principles and practice (prentice hall PTR New Jersey, 1996).

7. Sarabandi, K., Li, E. S. \& Nashashibi, A. Modeling and measurements of scattering from road surfaces at millimeter-wave frequencies. IEEE Transactions on Antennas and Propagation 45, 1679-1688 (1997).

8. Lamb, J. W. Miscellaneous data on materials for millimetre and submillimetre optics. International Journal of Infrared and Millimeter Waves 17, 1997-2034 (1996).

9. Etinger, A., Litvak, B. \& Pinhasi, Y. Multi ray model for near-ground millimeter wave radar. Sensors 17, 1983 (2017).

10. Constantine, A. B. et al. Antenna theory: analysis and design. MICROSTRIP ANTENNAS, third edition, John wiley $\mathcal{G}$ sons (2005).

11. Aragon-Zavala Alejandro ans Simon R, S. Antennas and propagation for wireless communication systems (John Wiley \& Sons, 2008).

12. Minghua, J., Guoxin, Z. \& Wenli, J. A new model for predicting the characteristic of $R F$ propagation in rectangular tunnel in Microwave Conference, 2008 China-Japan Joint (2008), 268-270.

13. Hrovat, A., Kandus, G. \& Javornik, T. A survey of radio propagation modeling for tunnels. IEEE Communications Surveys $\mathcal{E}$ Tutorials 16, 658-669 (2014).

14. Ghazal, A. et al. A non-stationary MIMO channel model for high-speed train communication systems in Vehicular Technology Conference (VTC Spring), 2012 IEEE 75th (2012), 1-5.

15. Liu, C., Zhang, E., Wu, Z. \& Zhang, B. Modelling radio wave propagation in tunnels with raytracing method in Antennas and Propagation (EuCAP), 2013 7th European Conference on (2013), 2317-2321.

16. Gan, M. et al. A ray tracing algorithm for intelligent transport systems in tunnels in Wireless Vehicular Communications (WiVeC), 2014 IEEE 6th International Symposium on (2014), 1-5. 